\title{
COMPARAISON DE DEUX MÉTHODES D'ESTIMATION DE LA PRODUCTION NATURELLE DE SMOLTS DE SAUMON ATLANTIQUE (SALMO SALAR L.)
}

\author{
J.L. BAGLINIĖRE (1), G. MAISSE (1), A. NIHOUARN (2), J.P.PORCHER (2), P.M. LE GLOANEC (1)
}

(1) Station de Physiologie et d'Écologie des poissons, I.N.R.A. - 35042 RENNES Cedex, France.

(2) Délégation régionale $n^{\circ} 2$ du Conseil Supérieur de la Pèche, 84, rue de rennes -35510 CESSONSÉVIGNE, France.

\section{RESUMÉ}

Deux méthodes d'estimation de la production en smolts de saumon atlantique (Salmo salar $L$.) sont comparées sur la rivière Oir, affluent de la Sélune (Basse-Normandie). La première estime le nombre de smolts par l'analyse des caractéristiques de l'habitat et du phénomène de bimodalité de taille observée dans la population $0+$ en automne. La seconde méthode évalue la production en smolts à partir des captures par piégeage.

L'étude montre que les estimations réalisées par les deux méthodes sont proches avec cependant des différences plus ou moins importantes selon l'année et la classe d'âge. Elle montre également que les smolts proviennent essentiellement de la population automnale de $0+$ à forte croissance. Enfin, les valeurs de taux de survie hivernale utilisées dans la première méthode permettent d'obtenir des estimations du nombre de smolts 1 - proches de celles faites par piègeage, les résultats sont moins fiables pour les smolts $2+$. Les résultats sont discutés et différents aspects sont analysés en vue d'améliorer la concordance entre les deux méthodes.

Mots-clé : Saumon atlantique, juvéniles, habitat, production, migration, rivière, France.

\section{COMPARISON OF TWO METHODS FOR THE ESTIMATION OF THE NATURAL PRODUCTION OF ATLANTIC SALMON SMOLTS (SaImo salar L.)}

\section{ABSTRACT}

Two estimations methods of smolts number are compared in Oir River, tributary of Selune River (Low-Normandy). The first one estimates the smolts number from the analysis of characteristics of the habitat and of growth bimodality phenomena observed in $0+$ autumn population. The second method evaluates the smolts production from the catches by trapping.

This study shows that the estimations made by the two methods are closed. Nervertheless there exits some more or less important differences according to year and age class. It shows also that the smolts come mainly from the upper mode of 0 + autumn population. Lastly, the over winter survival rates values used in the first method give $1+$ smolts estimations near of those made by trapping, results are less reliable for $2+$ smolts.

$R$ rsults are discussed and different aspects are analysed in order to obtain a better agreement between the two methods.

Key-words : Atlantic salmon, juveniles, habitat, production, migration, river, France.

\section{INTRODUCTION}

La connaissance de la production en smolts est utile à la gestion d'une population de saumon atlantique (Salmo salar L.). Actuellement, il existe deux méthodes pour quantifier la production en smolts des rivières françaises qui se caractérisent par la présence de deux classes d’âge à la smoltification $1+$ et $2+$ (PRÉVOST, 1987). La première est celle du piégeage des smolts lors de leur dévalaison. Pratiquée en France depuis 1948 (VIBERT, 1950), elle nécessite la connaissance de l'efficacité de piégeage selon les conditions de débit. La seconde est celle mise au point par BAGLINIERE et CHAMPIGNEULLE (1986). Cette méthode est fondée sur la caractérisation des peuplements automnaux de tacons en fonction des caractéristiques de l'habitat. Jusqu'à ce jour, ces deux méthodes n'avaient pas pu être comparées.

La mise en place d'un programme d'étude du saumon atlantique sur la rivière Oir, affluent de la Sélune (Basse-Normandie), où est installé un système de piégeage, a permis la comparaison de ces deux méthodes d'estimation. 


\section{TECHNIQUE ET MÉTHODES}

\subsection{Milieu étudié (Fig. 1)}

La Sélune, petit fleuve côtier de Normandie, forme avec la Sée un estuaire commun dans la baie du Mont Saint-Michel. Sa longueur est de $75 \mathrm{~km}$ pour une superficie de bassin versant de $1.010 \mathrm{~km}^{2}$; sa pente moyenne est de $1,9 \%$. Son embouchure présente une topographie variable du lit, due aux courants de marée. La présence du barrage infranchissable de "La Roche qui boit" limite l'accessibilité des géniteurs aux $16 \mathrm{~km}$ de la partie aval de la Sélune et à ses deux principaux affluents, l'Oir et le Beuvron.

L'Oir est un affluent rive droite de la Sélune; son confluent se situe à $8 \mathrm{~km}$ de l'estuaire. Son cours long de $19,5 \mathrm{~km}$ est orienté Est-Ouest et a une pente moyenne de $11 \%$. Son bassin versant, d'une étendue de $87 \mathrm{~km}^{2}$, est constitué de terrains schisteux comprenant quelques enclaves de granit ancien et est soumis à un climat océanique. La plupart des sols de ce bassin sont à vocation d'élevage extensif: $64 \%$ de la surface agricole utilisée sont en herbage et $23 \%$ en cultures fourragères (ANONYME, 1980).

Les résultats d'analyses physico-chimiques de l'eau effectuées sur l'Oir en 1980 et 1985 ont èté similaires à ceux d'études réalisées sur des rivières bretonnes (NISBET et VERNEAUX, 1970).

La zone de reproduction du saumon atlantique est répartie sur $11,5 \mathrm{~km}$ du cours de l'Oir et sur certains de ses affluents. Le moulin du Buat, toujours en activité, constitue un obstacle à la migration, son franchissement nécessitant des crues très importantes.

Un double système de piégeage (montée et descente) a été installé au moulin de Cerisel situé à $2,3 \mathrm{~km}$ du confluent de l'Oir et de la Sélune.

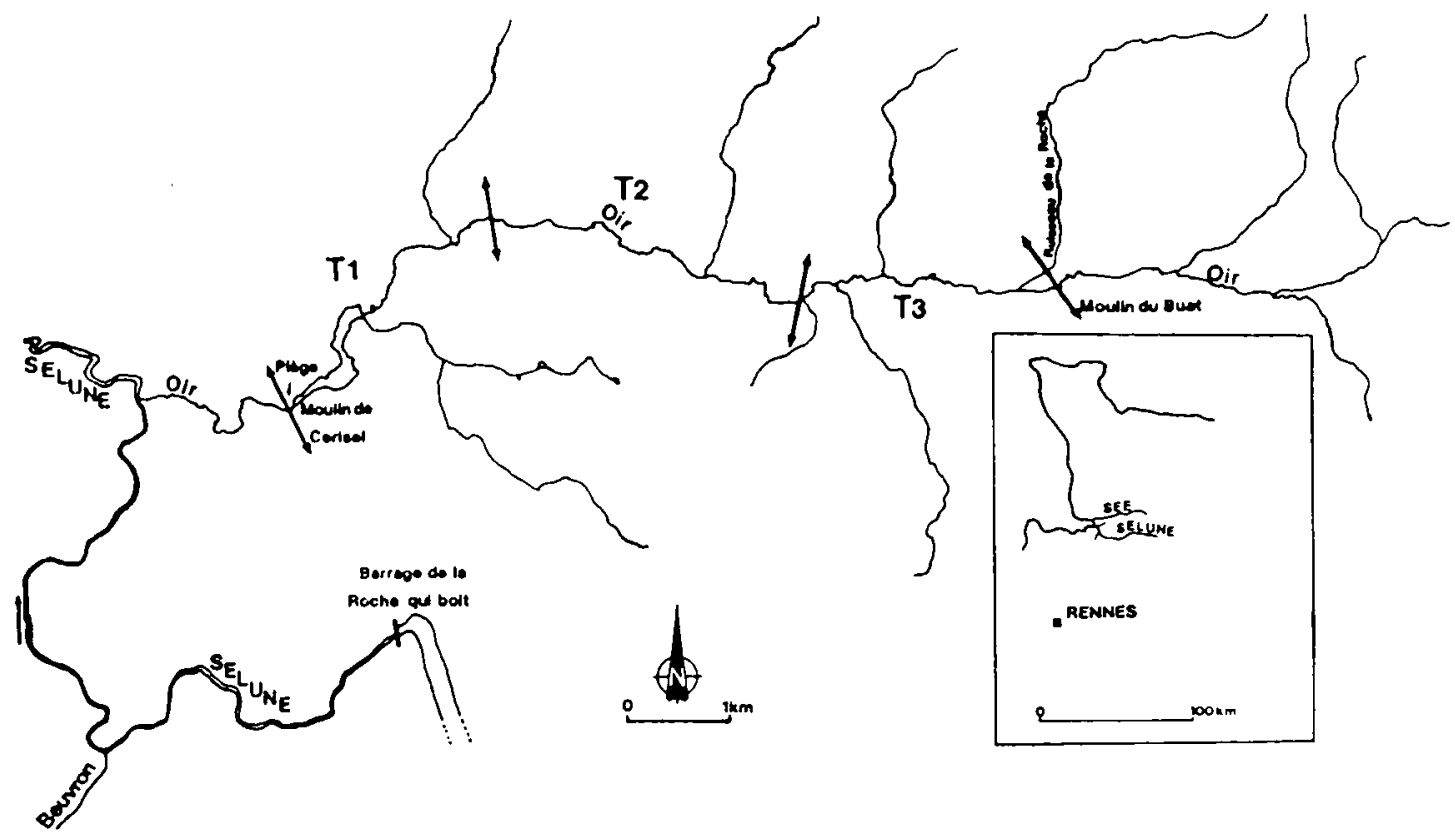

Figure 1 : Le bassin de l'Oir : situation des trois tronçons étudiés $\left(T_{1}, T_{2}, T_{3}\right)$

Figure 1 : The bassin of Oir River: situation of the three sections studied ( $\left.T_{1}, T_{2}, T_{3}\right)$.

\subsection{Récolte des données}

* Caractéristiques de l'habitat

L'habitat a été décrit en juillet 1985 sur toute la zone fréquentée par le saumon (à partir du moulin de Cerisel) en utilisant les descripteurs proposés par BAGLINIERE et CHAMPIGNEULLE (1986) : profondeur d'eau, vitesse de courant, nature du substrat. Quatre types de milieux bien distincts ont été définis à partir de ces paramètres (Tableau I) : radier, plat courant, plat et profond. La rivière a été divisée en trois tronçons en raison d'une répartition non uniforme de ces types d'habitats (Tableau II ; Fig. 1).

"Population de juvéniles

La population en place a été recensée par pêche électrique (deux passages; méthode de DELURY) en octobre 1985 sur deux secteurs representatifs d'un type d'habitat dans chaque tronçon et en octobre 1986 sur l'ensemble du cours colonisé par le saumon y compris le ruisseau de la roche (Fig. 1). 


\begin{tabular}{|c|c|c|c|c|c|c|c|c|c|}
\hline Type d'habitat & $\begin{array}{l}: \\
:\end{array}$ & $\begin{array}{l}\text { Profiondeur } \\
(\mathrm{cm})\end{array}$ & $\begin{array}{l}: \\
:\end{array}$ & $\begin{array}{l}\text { Vitesse de } \\
\text { courant } \\
(\mathrm{cm} / \mathrm{s})\end{array}$ & : & $\begin{array}{c}\text { Substrat } \\
(\star)\end{array}$ & & : & $\begin{array}{l}\text { Végétation } \\
\text { aquatique }\end{array}$ \\
\hline & : & & : & & : & & & : & \\
\hline Profonds & : & $>60$ & : & $<20$ & : & Type 1,2 ou & 3 & : & absence \\
\hline Plats & : & $>40$ & : & $20<v<30$ & $\begin{array}{l}: \\
:\end{array}$ & Type 1, 2 ou & 3 & : & absence \\
\hline Plats courants & $\begin{array}{l}: \\
:\end{array}$ & $<40$ & : & $30<V<40$ & : & Type 2 ou 3 & 3 & : & présence \\
\hline Radier & : & $<25$ & : & $>40$ & : & Type 2 ou 3 & 3 & : & présence \\
\hline
\end{tabular}

Tableau I : Caractéristiques des divers types d'habitat rencontrés sur l'Oir en période d'étiage.

Table I : Characteristics of different types of habitat present in River Oir in low water period.

(*) Type 1 : sableux uniforme

Type 2 : sableux mixte : plus de $60 \%$ de sédiments fins couvrent la surface du fond

Type 3 : grossier : plus de $40 \%$ d'éléments grossiers (> $2 \mathrm{~cm}$ ) couvrent la surface du fond

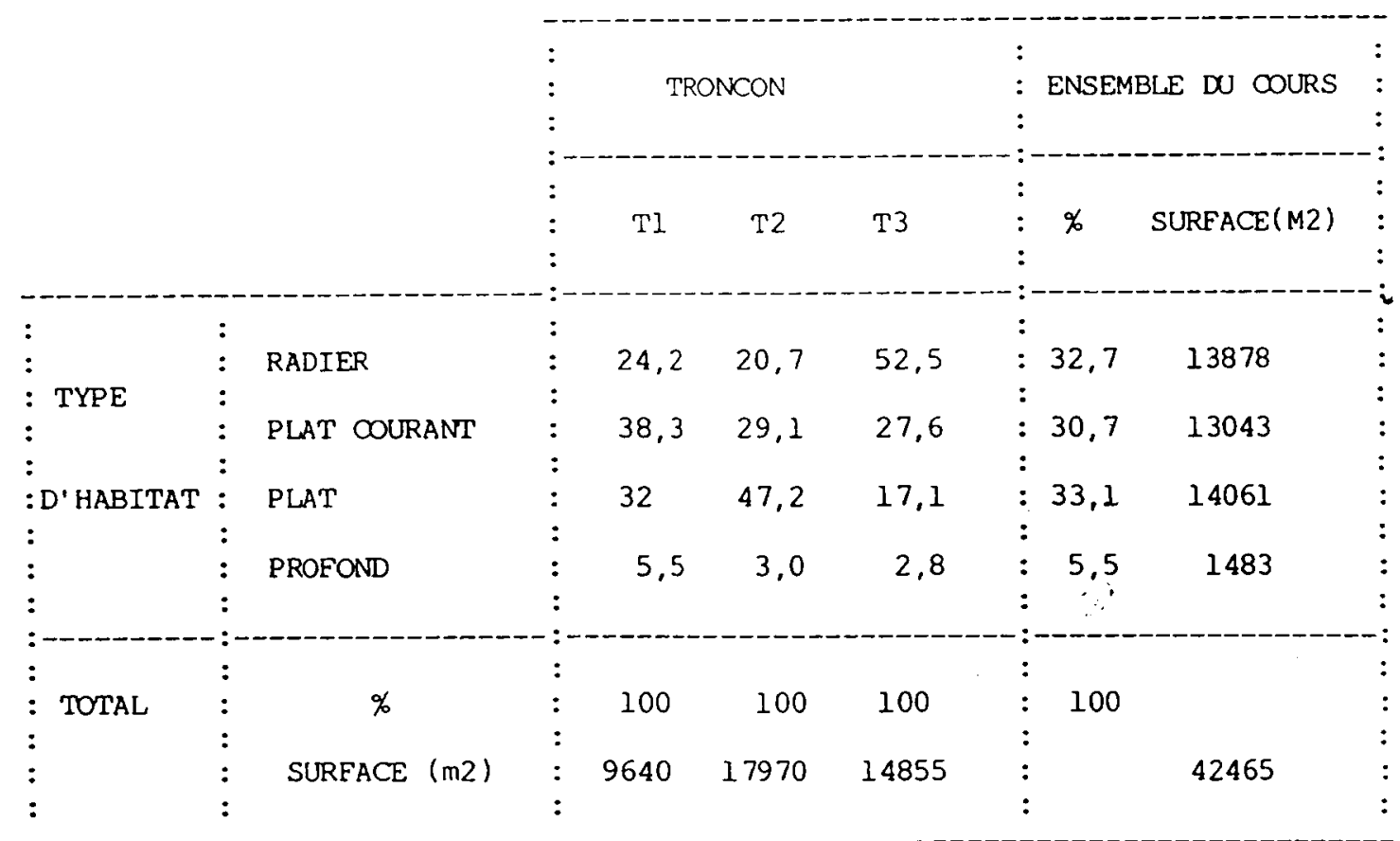

Tableau II : Distribution par tronçon (en pourcentage) des quatre types d'habitat rencontrés sur l'Oir.

Table II : Distribution (in percentage) in each section of the four types of habitat met in the Oir River

La population migrante a été recensée par piégeage continu de janvier à mai en 1986 et 1987.

Quelque soit le mode de capture, tous les poissons ont été mesurés (longueur fourche en $\mathrm{mm}$ ) et des écailles ont été prélevées sur la quasi-totalité des individus de taille supérieure ou égale à $100 \mathrm{~mm}$. Les mâles spermiants ont été détectés par pression des flancs. Lors de l'inventaire 1985, tous les juvéniles de l'année ont été tatoués au bleu alcian sur nageoire pectorale droite ou gauche selon un seuil de taille $(90 \mathrm{~mm})$ retenu à partir des résultats obtenus sur le Scorff par BAGLINIERE et CHAMPIGNEULLE (1986). Lors de la dévalaison, le stade (tacon, pré-smolt, smolt) a été défini à partir des critères classiques de morphologie et de couleur (JOHNSTON et EALES, 1967). 


\subsection{Traitement des données : estimation de la production en smolts}

\subsubsection{Mèthode de BAGLINIĖRE et CHAMPIGNEULLE (1986)}

Cette méthode est fondée sur trois points

a) Estimation des densités automnales des peuplements en tacons $0+$ et 1 + dans des secteurs représentatifs des différents habitats. Le milieu profond n'a pas été pris en compte en raison de la quasi-absence de juvéniles (BAGLINIËRE et CHAMPIGNEULLE, 1982; BAGLINIERE et ARRIBE MOUTOUNET, 1985). Les densités de population ont été estimées par la formule de SEBER et LECREN (1967) et la structure d'âge a èté dèterminée par scalimétrie (BAGLINIERE, 1979).

b) Analyse de l'histogramme de fréquence de taille des tacons $0_{-}$: la mise en évidence d'une éventuelle bimodalité a été réalisée par la méthode de BATTACHARYA (LAURENT et MOREAU, 1974). Par analogie avec ce qui a été décrit par THORPE et al. (1980), les individus du mode haut sont supposés smoltifier au printemps suivant, ceux du mode bas restant au moins encore une année en rivière.

c) L'estimation des mortalités hivernales suivant l'âge et la taille. En l'absence de données propres à l'Oir, la valeur du taux de survie hivernale est celle retenue par BAGLINIERE et CHAMPIGNEULLE (1986): $65 \%$ pour les $0+$ et $80 \%$ pour les 1 .

Le nombre estimé $(N)$ de smolts $1+$ produits par chaque type d'habitat est donné par la formule suivante :

$N=d \times A \times p(m \times s m+(1-m) s i m)$ où

$\mathrm{d}=$ densité automnale de $0-\left(\right.$ en ind $\left./ \mathrm{m}^{2}\right)$

$A=$ surface de l'habitat (en $\mathrm{m}^{2}$ )

$p=$ proportion d'individus $0+$ dans le mode haut

$\mathrm{m}=$ proportion d'individus spermiants dans le mode haut

$\mathrm{sm}=$ taux de survie hivernale des mâles spermiants

sim = taux de survie hivernale des immatures.

D'après MYERS (1984), la survie hivernale des mâles spermiants est deux fois plus faible que celle des immatures, d'où la formule simplifiée:

$N=d \times A \times p \times \operatorname{sim}(1-m / 2)$.

La même formule est appliquée aux individus automnaux $1+$ pour l'estimation de la production en smolts $2+$; dans ce cas la valeur de p est très proche de 1, puisque la présence de tacons $2+$ s'est avérée extrêmement faible, et $\mathrm{m}$ est la proportion d'individus spermiants observée dans l'échantillon.

La production totale peut alors être estimée de deux façons différentes:

- soit en additionnant la production de chacun des trois tronçons calculée à partir des densités par type d'habitat et des bimodalités propres à chaque tronçon

- soit en calculant directement la production de l'ensemble du cours d'eau à partir des valeurs moyennes des paramètres.

Enfin en 1986, l'estimation a pu être faite à partir de l'inventaire de la totalité du cours d'eau. Dans ce dernier cas, la bimodalité de taille mise en évidence sur le terrain par secteur (plusieurs secteurs formant un tronçon) a été utilisée.

\subsubsection{Méthode par piégeage des smolts}

L'efficacité du piégeage a été évaluée chaque année par la technique de marquagerecapture, pratiquée dans différentes conditions de débit.

Le nombre de smolts $(\mathrm{N})$ a été estimé par la formule suivante

ni

$N=\Sigma-\quad$ où

ei

$\mathrm{ni}=$ nombre de smolts piégés pour une période correspondant au débit i

ei $=$ efficacité de piégeage pour le débit i

Pour les deux méthodes, l'estimation a été calculée en tenant compte de l'intervalle de confiance (à $95 \%$ ) de chaque proportion (bimodalité, maturation).

\section{RÉsultats}

\subsection{Population en place}

La densité de la population a varié selon l'habitat, la classe d'âge et l'année de 0 à 22,6 ind $/ 100 \mathrm{~m}^{2}$ (Tableau III). En 1986, celle des saumons $0+(62 \%$ de la population totale) était en moyenne trois fois plus faible qu'en 1985 ( $89 \%$ de la population totale) alors que les densités des individus $1+$ restaient voisines. Quelle que soit l'année, près de $80 \%$ des effectifs ont été recensés sur l'habitat radier. 


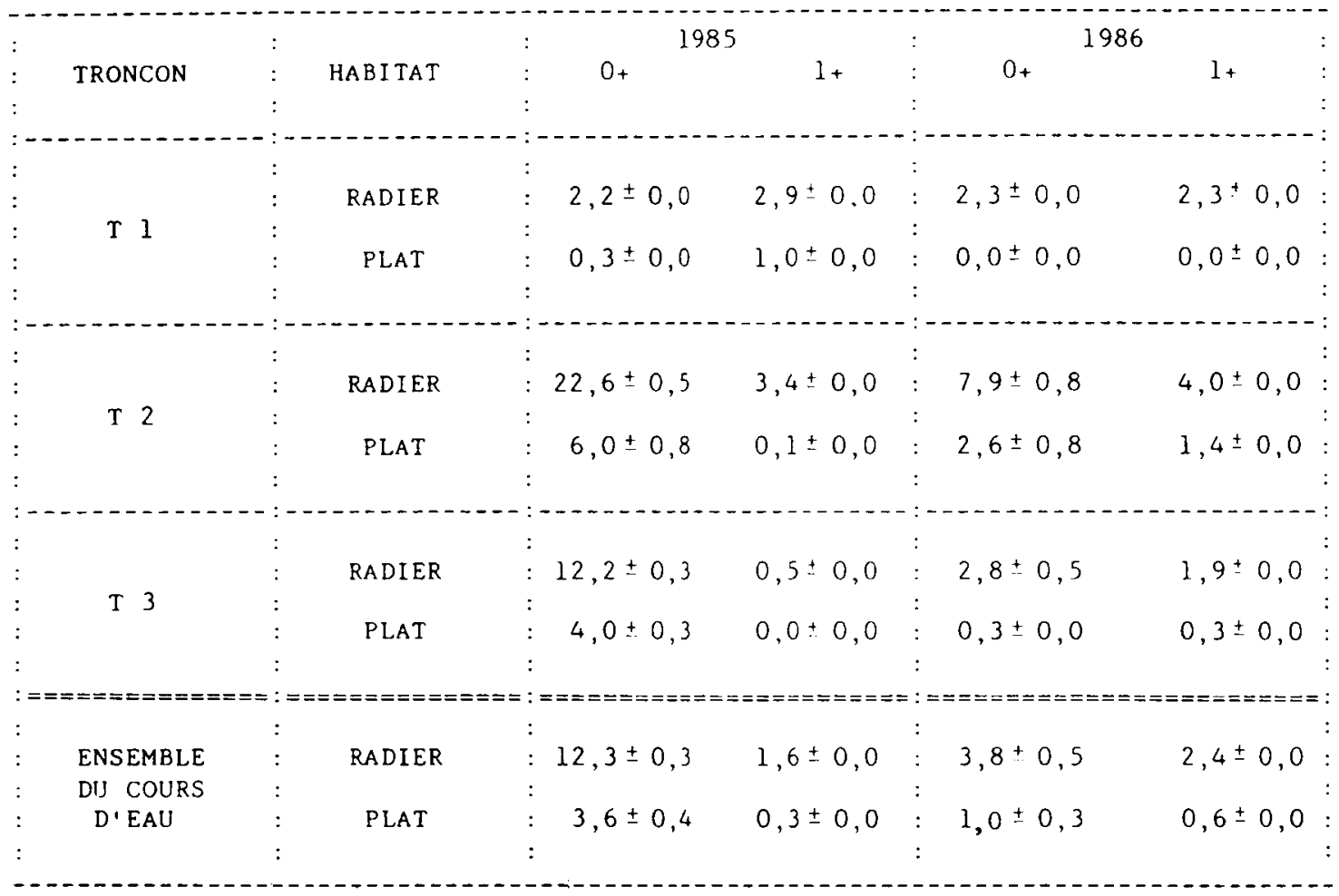

Tableau III : Densité (ind/100 $\mathrm{m}^{2}$ ) des juvéniles de saumon 0 + et 1 + estimée par type d'habitat. Table III : Density (ind/100 $\mathrm{m}^{2}$ ) of Salmon $0+$ and $1+$ juveniles estimated for each type of habitat.

En 1985, la taille moyenne des deux classes d'âge était significativement plus élevée $(p<0,01)^{*}$ qu'en 1986 (Tableau IV). Chaque année, la population $0+$ de chaque tronçon présentait une bimodalité de taille avec une majorité d'individus appartenant au mode haut (sauf dans la zone 3 en 1986). Cependant, l'écart de la taille entre ces deux modes était plus important en 1985 en raison du meilleur taux de croissance.

La proportion d'individus $1+$ spermiants était importante et supérieure à $50 \%$ chaque année. Par contre celle des individus $0+$ était beaucoup plus faible et a varié annuellement. Les saumons spermiants de cette classe d'âge ont toujours été répartis entre le haut du mode bas et le bas du mode haut. Quelle que soit l'année, il n'est apparu aucune différence significative entre les proportions des individus maturants des deux classes d'âge selon le tronçon (Tableau V).

\subsection{Population migrante}

888 saumons ont été piégés lors de la dévalaison de 1986, et 283 durant celle de 1987. Lors de la première année, $96 \%$ de la population dévalante était âgée d' $1+$ an alors que la répartition des deux classes était équilibrée l'année suivante. En 1986, la taille moyenne des deux classes d'âge de migrants était significativement plus élevée $(p<0,001)$ qu'en 1987.

En 1986, la dévalaison a été plus longue et plus précoce (2 janvier au 15 mai) qu'en 1987 (14 février au 30 avril) mais sa date mediane ( $50 \%$ des captures) a été plus tardive (21 avril contre le 9 avril). Chaque année, on a observé d'abord une descente de tacons puis de pré-smolts et enfin de smolts.

Cependant, la répartition annuelle des différents stades était significativement différente $(p<0,001)$ en raison d'une très forte proportion de smolts en 1987 et d'une distribution équilibrée entre tacons et smolts en 1986 (Tableau VI). Quelle que soit l'année, les $2+$ ont dévalé en moyenne plus tôt que les $1+$.

Lors de la dévalaison de 1986, 39 individus marqués en 0+ l'automne précédent ont été recapturés. Ces recaptures provenaient significativement plus $(p=0,01)$ des poissons ayant une taille en $0+$ supérieure ou égale à $90 \mathrm{~mm}(37 / 178$ contre $2 / 78)$. 


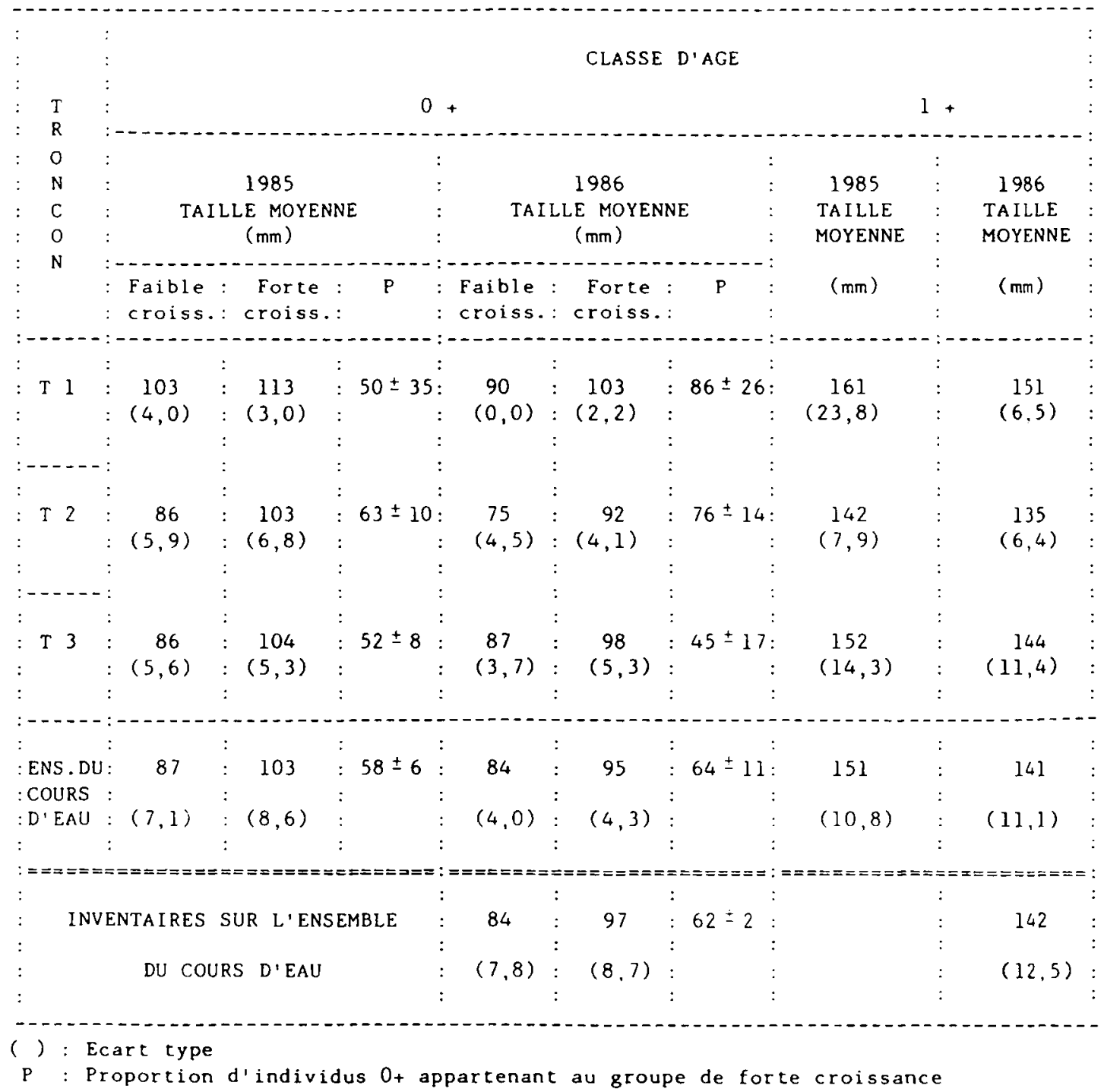

Tableau IV : Bimodalité de tallle dans la population de saumon $0+$ et talle moyenne des individus 1 - echantillonnés sur l'Oir en 1985 et 1986.

Table IV : Bimodality of length in the $0+$ Salmon population and mean length of $1+$ individuals sampled in the Oir River in 1985 and 1986.

\subsection{Estimation de la production en smolts}

Selon l'année, l'estimation du nombre de smolts effectuée, en tenant compte des caractéristiques automnales des populations par tronçon, a été identique et supérieure à celle réalisée en considérant les caractéristiques moyennes de l'ensemble de la rivière (Tableau VII).

En 1986, le nombre de smolts $1+$ estimé à partir des caractéristiques des peuplements par tronçon était proche de celui évalué par piégeage. Par contre les estimations étaient très éloignées pour l'autre classe d'âge.

En 1987, quelles que soient les méthodes utilisant les caractéristiques des populations automnales, elles ont donné des estimations des deux classes d'âge de smolts très supérieures à celles évaluées par piégeage. En revanche, l'évaluation du nombre de smolts réalisée à partir de l'inventaire automnal de l'ensemble du cours d'eau a fourni des résultats identiques à ceux obtenus par piégeage. Les estimations du nombre de smolts $2+$ étaient un peu moins proches.

L'évaluation du nombre de smolts fournis par tronçon a montré pour chacune des années l'importance du tronçon 2 dans la production totale de la rivière. (Tableau VIII). 


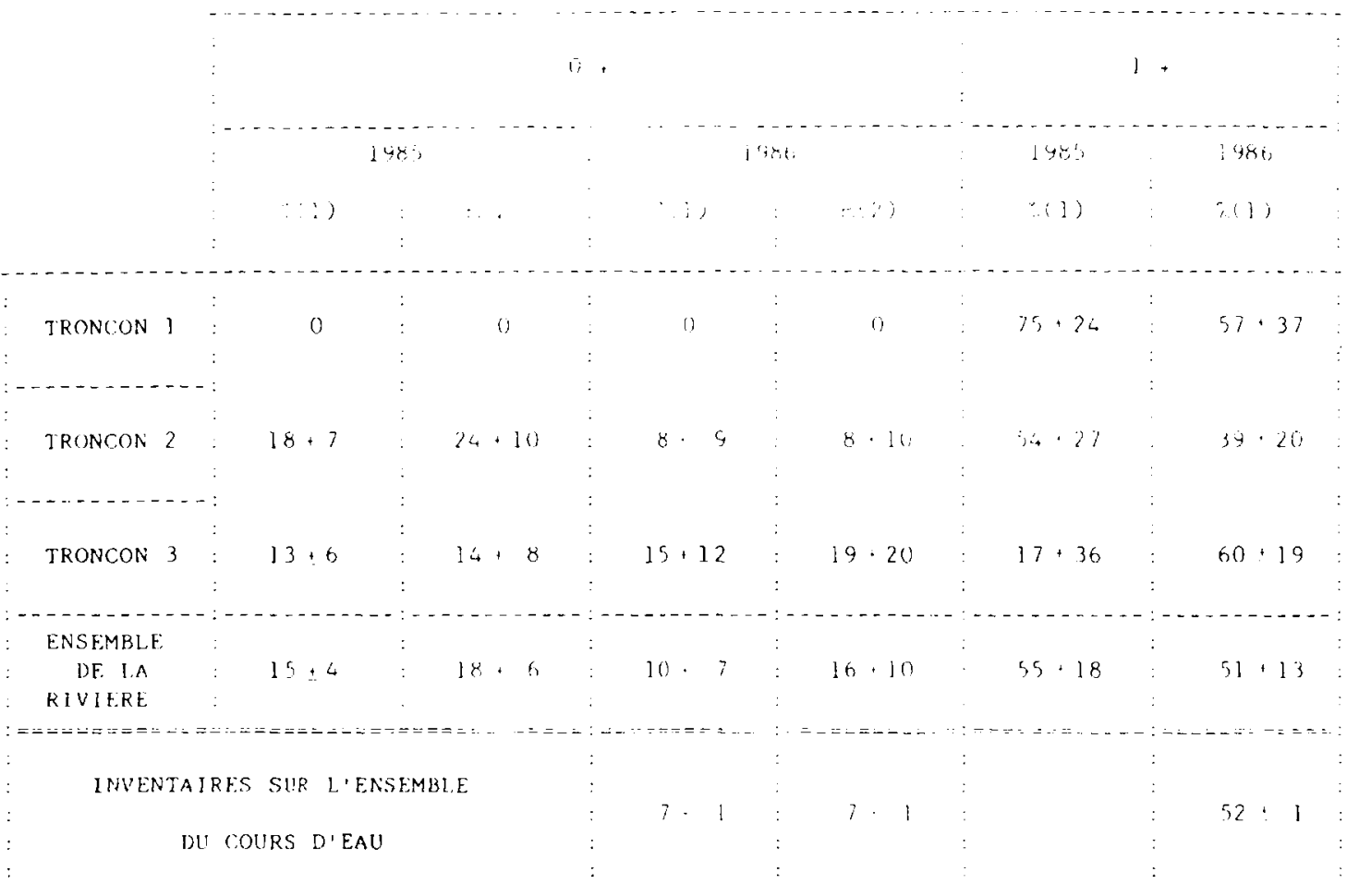

Tableau V : Pourcentage d'individus spermiants $0+$ et $1+$ dans la population de saumon atlantique échantillonnée sur l'Oir en 1985 et 1986.

Table $\mathrm{V}$ : Percentage of milting $0+$ and $1+$ individuals in the population of atlantic Salmon sampled in the Oir River in 1985 and 1986

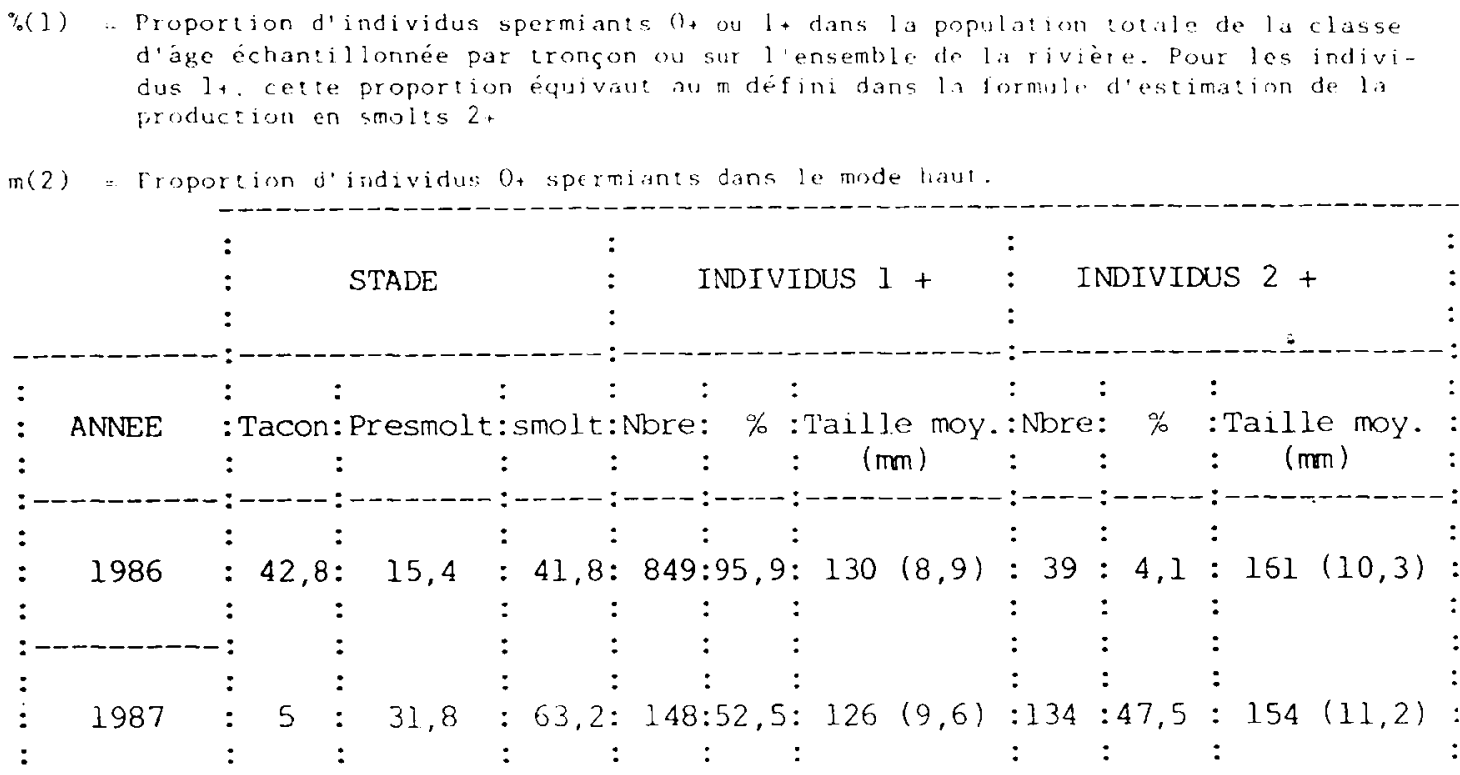

( ) : Ecart-type

Tableau VI : Nombre et caractéristiques des juvéniles de saumon $1+$ et 2 + capturés par piégeage sur l'Oir en 1986 et 1987.

Table VI : Number and characteristics of $1+$ and $2+$ juvenile Salmons trapped in the Oir River in 1986 and 1987. 


\section{DISCUSSION}

Les résultats obtenus dans cette étude ont permis de vérifier dans les conditions naturelles que les saumons $0+$ du mode haut smoltifient le printemps suivant. En effet, la faible erreur d'appartenance au groupe de forte croissance (75\% en 1985) due à la taille limite intermodale imposée $(90 \mathrm{~mm})$ et le pourcentage elevé $(95 \%)$ d'individus 1 + recapturés, marqués comme $0+$ à une taille supérieure ou égale à $90 \mathrm{~mm}$, confirment bien que les smolts $1+$ proviennent essentiellement du mode haut. Cependant la recapture de deux individus 1 + marqués en $0+$ à une taille inférieure à $90 \mathrm{~mm}$ laisserait supposer une possible mais très faible participation du groupe de faible croissance à la production en smolts 1 + à moins que ces individus n'aient appartenu à la population $0+$ d'un secteur amont du tronçon 3 où la croissance est plus faible.

\begin{tabular}{|c|c|c|c|c|c|c|c|c|c|c|}
\hline : & & : & : & & & & : & & & \\
\hline : & & : & : & NOMBRE DE & SMOLTS & F.STIME & : & & & \\
\hline : & & : & : & & & & $:$ & & & \\
\hline : & ANNEE & METHODE & $:-$ & $\ldots \ldots \ldots$ & $-\cdots--$ & --------- & : & TOTA & & \\
\hline : & & : & : & & : & & $:$ & & & \\
\hline : & & : & : & $1+$ & : & $2+$ & : & & & \\
\hline : & & $:$ & $:$ & & : & & : & & & : \\
\hline & -- & 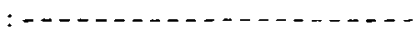 & $:-$ & ------- & $--:--$ & $-----\cdots$ & $:-$ & ----- & $\cdots$ & \\
\hline : & & : & $:$ & & $:$ & & : & & & \\
\hline : & & : Caractérisation de & : & $1019 \pm 298$ & : & $182 \pm 33$ & : & $1201 \pm$ & 270 & \\
\hline : & & : l'habitat par tronçon & $:$ & & : & & $:$ & & & \\
\hline : & & $:$ & $:$ & & $:$ & & : & & & \\
\hline : & & : - - - - - - & & & : & & : & & & \\
\hline : & 1 & $:$ & : & & : & & $:$ & & & \\
\hline : & 9 & : Caractérisation de & $:$ & & : & & : & & & \\
\hline : & 8 & : l'habitat sur l'ens. & $:$ & $924 \pm 180$ & : & $176 \pm 22$ & $:$ & $1100 \pm$ & 147 & \\
\hline : & 6 & : du cours d'eau & $:$ & & : & & : & & & : \\
\hline : & & $:$ & $:$ & & $:$ & & $:$ & & & : \\
\hline : & & 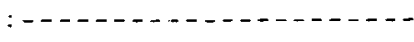 & & & : & & : & & & : \\
\hline : & & $:$ & $:$ & & : & & $:$ & & & : \\
\hline : & & Piégeage & : & $1270 \pm 181$ & $:$ & $55 \pm 8$ & $:$ & $1325 \pm$ & 189 & : \\
\hline : & & : & : & & : & & : & & & : \\
\hline & ---1 & 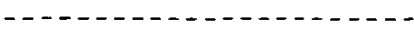 & - & $----n---1$ & -- & $--\ldots-n$ & - & ----- & --- & \\
\hline : & & : & $:$ & & : & & : & & & : \\
\hline : & & : Caractérisation de & : & $397+185$ & $:$ & $343 \pm 48$ & $:$ & $740+$ & 158 & : \\
\hline : & & : l'habitat par tronçon & $:$ & & $:$ & & $:$ & & & \\
\hline : & & $:$ & $:$ & & $:$ & & $:$ & & & \\
\hline : & & : & $:$ & & : & & : & & & : \\
\hline : & & $:$ & $:$ & & : & & $:$ & & & \\
\hline : & & : Caractérisation de & $:$ & & : & & : & & & \\
\hline : & 1 & : l'habitat sur l'ens. & $:$ & $308+103$ & : & $295 \pm 26$ & : & $503 \pm$ & 95 & \\
\hline : & 9 & : du cours d'eau & $:$ & & : & & : & & & \\
\hline : & 8 & $:$ & $:$ & & $:$ & & : & & & \\
\hline : & 7 & $:-\ldots+\ldots+\ldots, \ldots+\ldots$ & $:$ & & : & & : & & & \\
\hline : & & $:$ & $:$ & & : & & : & & & \\
\hline : & & : Inventaire de l'ens. & $:$ & $191 \pm 11$ & $:$ & $232 \pm 2$ & : & 423 & +9 & \\
\hline : & & : du cours d'eau & : & & : & & : & & & \\
\hline : & & $:$ & $:$ & & : & & : & & & \\
\hline : & & 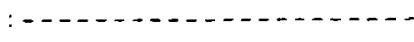 & $:$ & & : & & $:$ & & & \\
\hline : & & $:$ & $:$ & & : & & $:$ & & & \\
\hline : & & Piégeage & : & $199 \pm 37$ & : & $180 \pm 33$ & : & 379 & 70 & \\
\hline : & & : & : & & : & & : & & & \\
\hline
\end{tabular}

Tableau VII : Estimation de la production en smolts de saumon atlantique sur l'Oir en 1986 et 1987.

Table VII : Estimation of the production of smolts of atlantic Salmon in the Oir River in 1986 and 1987. 


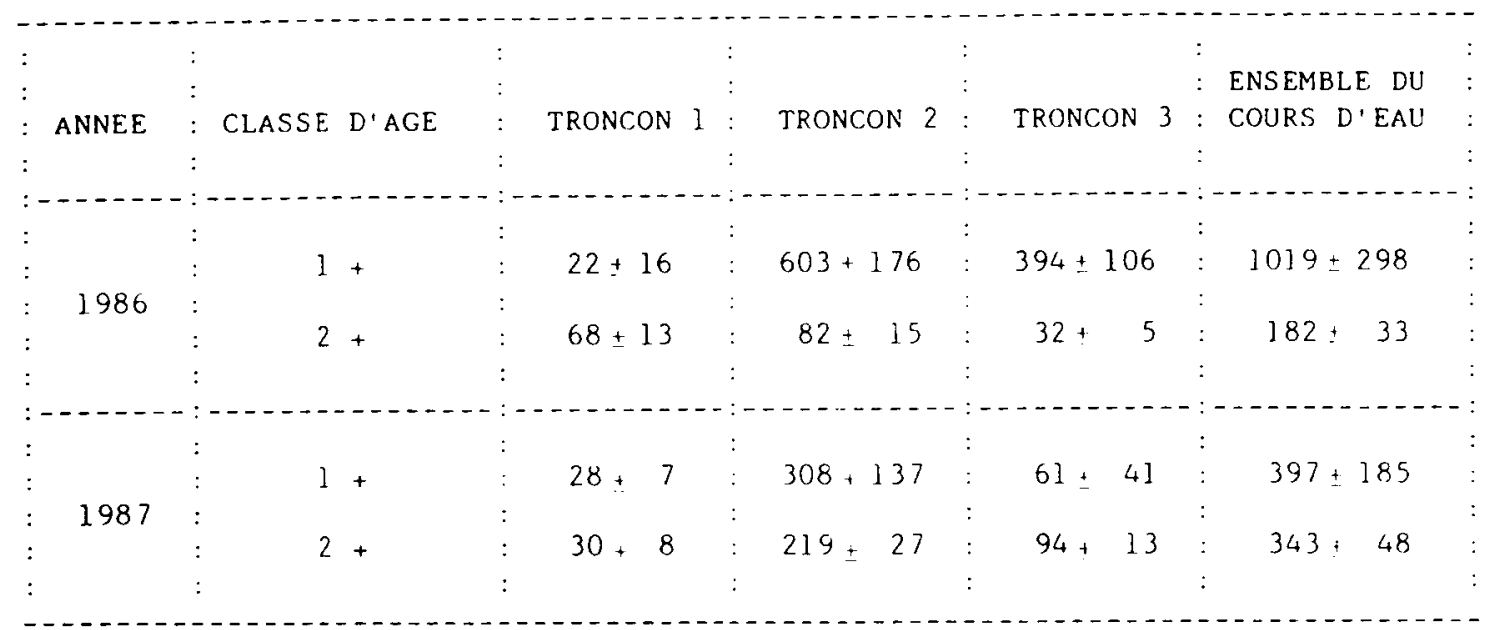

Tableau VIII : Nombre estimé de smolts 1+ et 2+ produits par tronçon sur l'Oir en 1986 et 1987. Table VIII : Estimated number of $1+$ and $2+$ smolts produced by each section of the Oir River in 1986 and 1987.

Les estimations du nombre de smolts effectuées par les deux méthodes peuvent différer selon l'année et la classe d'âge, des évaluations très proches étant réalisées pour les smolts $1+$. La comparaison des différentes estimations a permis d'analyser plusieurs aspects:

- la surface inventoriée et la représentativité des secteurs.

Le taux d'échantillonnage de la surface en eau est faible $(6,5 \%)$. Néanmoins il reste comparable à celui inventorié sur le Girnock Burn en Ecosse (7\%: BUCK et HAY, 1984) et est supérieur à celui analysé sur le Scorff (2,1\%: BAGLINIERE et CHAMPIGNEULLE, 1986) pour le même type d'étude. L'absence d'inventaires dans les milieux profonds ne modifie pas les estimations compte tenu de sa faible proportion dans la surface en eau (Tableau 9) et de la quasi-absence de saumons dans cet habitat lors de l'inventaire exhaustif de 1986. Par contre, les milieux plats ont été largement sous-échantillonnés par rapport aux habitats radiers alors qu'ils constituent plus des deux tiers de la surface en eau totale et plus de $76 \%$ ce celle du tronçon 2 qui est le plus productif en smolts. Notons que la surface calculée reste une estimation, dont il serait intéressant de connaître l'intervalle de confiance.

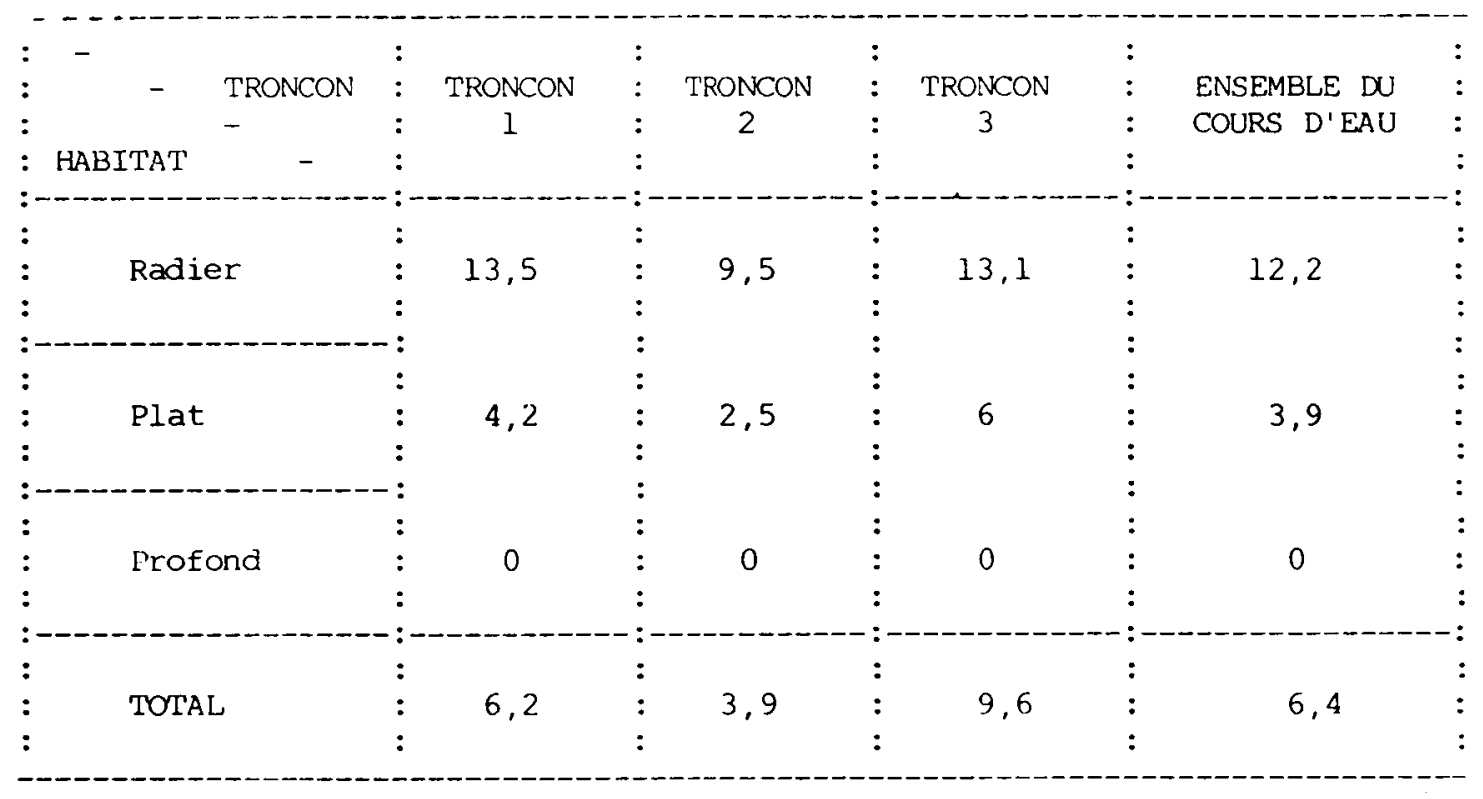

Tableau IX : Répartition (en \%) des surfaces d'habitat échantillonnées par tronçon.

Table $\mathrm{IX}$ : Distribution (in \%) of habitat areas sampled in each section. 
L'ensemble de ces considerations a pu revêtir une importance non négligeable dans le cas de l'Oir car la petite largueur et la faible pente de la rivière rendent plus difficiles la détermination d'habitats homogenes possédant une taille suffisante (répartition en mosaïque).

Enfin les affluents de l'Oir ont été peu étudiés alors que la densité en saumons sur les secteurs inventoriés y était supérieure ou égale à celle observée dans la rivière. Si, dans le cas du Scorff (bassin versant plus de cinq fois superieur à celui de l'Oir) les affluents jouent un rôle négligeable dans la production totale en smolts (BAGLINIERE et MAISSE, données non publiées), il n'en est certainement pas toujours de même sur l'Oir.

- le taux de survie hivernale

Au vu des résultats, les valeurs du taux de survie hivernale utilisées semblent à priori acceptables pour les deux classes d'âge. Cependant elles paraissent plus aléatoires pour les smolts $2+$. Par ailleurs, il est vraisemblable que ces valeurs doivent varier annuellement en fonction des conditions climatiques durant l'automne et l'hiver, de la densité automnale et de la proportion d'individus maturants. Ce dernier facteur joue certainement un rôle important dans la survie hivernale des smolts 2 - puisque des mortalités importantes liées à la maturation sexuelle dès l'âge de 2 ans ont souvent été constatées (OSTERDAHL, 1969; LEYZEROVICH, 1973; MITANS, 1973; DALLEY et al., 1983; MYERS, 1984).

\section{- l'efficacité du piège}

Deux expériences de marquage-recapture ont été réalisées lors de la dévalaison 1986 et une seule en 1987. Dans tous les cas, elles ont été effectuées avec des individus d'un an. Or en 1986, aucune expérience n'a été faite pendant la période de très forte crue (25 mars au 9 avril) au cours de laquelle $25 \%$ de la population totale de smolts 2 - est piégée. De plus $46 \%$ de ces individus ont été capturés en période d'augmentation du niveau d'eau. Ceci peut expliquer la sous-estimation de cette classe d'âge par piégeage en 1986.

\section{- le stade de descente}

Durant les deux périodes de dévalaison, une certaine proportion de jeunes saumons (pouvant aller jusqu'à $43 \%$ en 1986) descendent à l'état de tacon. En fait, la succession dans le temps de ces individus puis des pré-smolts et enfin des smolts laisse supposer que les parrs sont des saumons en voie de smoltification. De même sur le Girnock Burn, BUCK et HAY (1984) n'observent pas de saumons totalement smoltifiés lors de la dévalaison printanière; des juvéniles marqués et dévalant comme tacons en automne ont été recapturés par la suite comme adulte, montrant bien que ces juvéniles migrent en mer (YOUNGSON et al., 1983).

\section{CONCLUSION}

Cette étude a montré que les résultats obtenus par les méthodes d'estimation étaient proches, variant dans le même sens, avec cependant des différences plus ou moins importantes selon l'année et la classe d'âge.

La précision de l'estimation obtenue par la méthode de BAGLINIERE et CHAMPIGNEULLE (1986) dépend directement de la qualité de représentation des secteurs inventoriés par tronçon. Une étude plus précise des relations tacon-habitat apparaît nécessaire afin de mieux cerner les fluctuations en fonction de la densité.

La méthode d'estimation par piégeage nécessite la connaissance de l'efficacité de capture en fonction du débit, de l'état physiologique et de l'âge des poissons. En l'absence de ces données, le nombre de captures ne saurait être que minimum, l'estimation la plus probable restant inconnue.

La méthode de BAGLINIERE et CHAMPIGNEULLE (1986) semble particulièrement bien adaptée aux populations de saumon du Massif Armoricain en raison de la faible dimension des cours d'eau et du taux de smoltification en 1 + important (BAGLINIERE et al., 1987). Cette méthode présente l'avantage de limiter les interventions sur le terrain à quelques inventaires, contrairement au piégeage qui nécessite des installations coûteuses et une présence continuelle de personnel pendant les trois mois que dure en moyenne la descente.

\section{REMERCIEMENTS}

Ce travail a été réalisé dans le cadre d'une convention de Recherche INRA-CSP mise en place pour étudier la dynamique des salmonidés migrateurs de l'Oir.

Les inventaires ont été réalisés avec la participation des Gardes-Pêche de la Fédération de la Manche et de la Brigade Mobile d'Intervention du CSP.

La récolte des données de piégeage a été effectuée en collaboration avec R. DELANOE. 


\section{BIBLIOGRAPHIE}

ANONYME, 1980. Recensement général de l'Agriculture 1979 - 1980. Premiers résultats. Ministère de l'Agriculture. Direction Départementale de l'Agriculture. Services statistiques, 1 vol, $32 \mathrm{p}$.

BAGLINIERE J.L., 1979. Les principales populations de poissons sur une rivière à salmonidés de Bretagne Sud, le Scorff. Cybium, $7: 53-74$.

BAGLINIERE J.L., CHAMPIGNEULLE A., 1982. Densités des populations de Truite communes (Salmo trutta L.) et de juvéniles de Saumon atlantique (Salmo salar L.) sur le cours principal du Scorff (Bretagne). Préférendums physiques et variations annuelles (1976-1980). Acta Oecologica Oecol. applic., $3: 241-256$.

BAGLINIERE J.L., ARRIBE-MOUTOUNET D., 1985. Microrépartition des populations de truite commune (Salmo trutta L.) de juvéniles de Saumon atlantique (Salmo salar L.) et des autres espèces présentes dans la partie haute du Scorff (Bretagne). Hydrobiologia, 120: 229-239.

BAGLINIERE J.L., CHAMPIGNEULLE A., 1986. Populations estimates of juvenile Atlantic Salmon (Salmo salar) as indices of smolt production in the river Scorff, Brittany. J. Fish. Biol., 29 : 467-482.

BAGLINIERE J.L., PROUZET J.P., NIHOUARN A., et MAISSE G., 1987. Caractéristiques générales des populations de Saumon atlantique (Salmo salar L.) des rivières du Massif armoricain. In "La restauration des rivières à saumon" Colloque franco-québécois 28 mai-1er juin 1985 . Hydrobiologie et Aquaculture. 23-27

BUCK R.J.G., HAY D.W., 1984. The relation between stock size and progeny of Atlantic salmon (Salmo salar L.) in a scottish stream. J. Fish. Biol., $23: 1-11$.

DALLEY E.L., ANDREWS C.W., GREEN J.M., 1983. Precocious male Atlantic salmon in insular Newfoundland. Can J. Fish. Aquat. Sci., 40:647-652.

JOHNSTON C.E., EALES J.G., 1967. Purines in the Integument of Atlantic salmon (Salmo salar) during parr-smolt transformation. J. Fish. Res. Bd. Canada, 24 : 955-964.

LAURENT M., MOREAU G., 1973. Influence des facteurs écologiques sur le coefficient de condition d'un téléostéen (Cottus gobio L.). Ann. Hydrobiol., 4 : 211-228.

LEYZEROVICH K.A., 1973. Dwarf males in hatchery propagation of Atlantic salmon J. Ichthyol., 13: 382-392.

MYERS R.A., 1984. Demographic consequences of precocious maturation of Atlantic salmon (Salmo salar). Can. J. Fish. Aquat. Sci., $41: 1349-1353$.

NISBET M., VERNEAUX J., 1970. Composantes chimiques des eaux courantes. Discussion et proposition de classe en tant que bases d'interprétation des analyses chimiques. Ann. Limn., 6 : 164-190.

PREVOST E., 1987. Les populations de Saumon atlantique (Salmo salar L.) en France. Description. Relation avec les caractéristiques des rivières. Essai de discrimination. Thèse Doct. Ingénieur. Sci. Agronom. ENSA, Rennes, 1 vol., 103 p.

OSTERDAHL L., 1969. The smolt run of a small swedish river. In Symposium on Salmon and Trout in streams. H.R. Mac Millan lectures in Fisheries, Univ. of British Colombia, Vancouver, Canada, PP : 205-215.

SEBER G.A.F., LE GREN E.D., 1967. Estimating population parameters from catches large relative to the population. J. Anim. Ecol., $36: 631-643$.

THORPE J.E., MORGAN R.I.G., OTTAWAY E.M., MILES M.S., 1980. Time of divergence of growth groups between potential $1+$ and $2+$ smolts among sibling salmon. J. Fish. Biol., 17: 13-21.

VIBERT R., 1950. Recherches sur le saumon de l'Adour (Salmo salar L.) (Ages, croissance, cycle génétique, races), 1942-1948. Ann. St. Cent. Hydro. appl., 3 : 27-148.

YOUNGSON A.F., BUCK R.J.G., SIMPSON T.H., HAY D.W., 1983. The autumn and spring emigrations of juvenile Atlantic salmon (Salmo salar L.), from the Girnock Burn, Aberdeenshine, Scotland: environmental release migration. J. Fish. Biol., $23: 625-639$. 